\title{
TTR
}

Traduction, terminologie, re?daction

\section{Annie Brisset, Sociocritique de la traduction : Théâtre et altérité au Québec (1968-1988). Longueuil, Le préambule, 1990, 347 p. ISBN 2-89133-114-1}

\section{Jane Koustas}

Volume 4, numéro 1, 1st semester 1991

Languages and Cultures in Translation Theories

URI : https://id.erudit.org/iderudit/014736ar

DOI : https://doi.org/10.7202/014736ar

Aller au sommaire du numéro

Éditeur(s)

Association canadienne de traductologie

ISSN

0835-8443 (imprimé)

1708-2188 (numérique)

Découvrir la revue

Citer ce compte rendu

Koustas, J. (1991). Compte rendu de [Annie Brisset, Sociocritique de la traduction : Théâtre et altérité au Québec (1968-1988). Longueuil, Le préambule, 1990, 347 p. ISBN 2-89133-114-1]. TTR, 4(1), 158-163.

https://doi.org/10.7202/014736ar d'utilisation que vous pouvez consulter en ligne.

https://apropos.erudit.org/fr/usagers/politique-dutilisation/ 
franciscain anglais répugne au néologisme. Estimant qu'il est impossible de saisir la pensée d'un auteur en traduction, Bacon préconise l'étude des langues savantes: hébreu, grec, arabe, araméen. $\mathrm{La}$ recherche d'une langue universelle aura comme étape préliminaire la constitution de thesauri linguarum, de lexiques, glossaires et tables de correspondance, et l'élaboration de méthodes comparatistes. Elle profitera paradoxalement à la réflexion sur la traduction en révélant la complexité des problèmes.

La lecture de ce recueil, à mener en parallèle avec celles de The Theory and Practice of Translation in the Middle Ages (Roger Ellis, dir.) et Medieval Translators and their Craft (Jeanette Beer, dir.) parus la même année, soulève des questions passionnantes. Questions d'histoire intellectuelle: le savoir médiéval a été tributaire en premier lieu des aléas de la tradition manuscrite des originaux latins, grecs, arabes, en second lieu de la disponibilité et de la qualité des traductions. Questions culturelles et politiques: Quel est le statut social du traducteur? Savant, auteur ou simple intermédiaire? Que devient l'outil de la traduction entre les mains des cultures fortes et des cultures faibles? Comment culture orale et culture écrite s'articulentelles dans la problématique de la traduction? Questions littéraires et textuelles: Dépendance du traducteur à l'égard de modèles littéraires de la culture-cible, dynamique entre le texte et sa traduction; la glose, qui naît de l'incapacité du traducteur à restituer le sens et qui s'amplifie jusqu'à se détacher et devenir œuvre indépendante, se révèle un phénomène central de la problématique de la traduction au moyen âge.

\author{
Claire le Brun \\ Université Concordia
}

\begin{abstract}
Annie Brisset, Sociocritique de la traduction: Théâtre et altérité au Québec (1968-1988). Longueuil, Le Préambule, 1990, 347 p. ISBN 2-89133-114-1
\end{abstract}

Si Edward Blodgett a pu demander aux lecteurs et aux traducteurs de la littérature québécoise «How Do You Say Gabrielle Roy?», Annie Brisset ne se sent nullement obligée de poser la question. Les auteurs en traduction qu'elle traite dans son livre, Brecht et Shakespeare entre 
autres, n'auraient pas à s'inquiéter de la mauvaise prononciation de leur nom: on ne parlerait probablement pas d'eux et, de toute façon, sûrement pas à haute voix. L'Autre de la langue source se trouve occulté ou éclipsé quand on essaie d'insérer dans le discours de la société québécoise le discours porté par les textes étrangers. C'est sur le rôle du traducteur dans cette opération qu'A. Brisset s'interroge dans cet excellent livre d'une originalité qu'il faut souligner. Elle se propose d'étudier le traducteur non pas comme «un alchimiste de langue», selon le mot de Jean Delisle, ni comme un artiste, scientifique ou artisan comme le suggèrent d'autres auteurs. Le traducteur ici est plutôt porte-parole d'un discours social et participant à un projet social, culturel et politique qui dépasse celui de la simple traduction d'une œuvre étrangère. Comme le signale Antoine Berman dans la préface, cette étude se constitue donc en une analyse poétique, éthique, historique et sociologique dont le titre en résume bien le projet. Il ne s'agit pas dans cette sociocritique de la traduction d'une réouverture du débat entre les traducteurs «assimilateurs» et ceux qui veulent respecter et conserver la différence. La question se pose autrement: comment le traducteur traduit-il quand il cherche à intégrer le texte de l'Autre dans son propre discours social, projet qui rejoint, dans le cas des traducteurs discutés, celui de la fondation d'un théâtre national québécois auquel est liée l'existence même de la future nation? Comment et pourquoi «québéciser les originaux pour les annexer et créer une langue» (p. 13)?

Ayant établi la direction de son étude dans l'introduction, l'auteure s'attache ensuite à en définir et à en justifier les limites. Son analyse porte d'une part sur les textes dramatiques, puisque le théâtre est le genre le plus traduit, et d'autre part sur les traductions thêâtrales publiées ou représentées au Québec entre 1968 et 1988, c'est-à-dire à partir du moment où l'on parle d'un véritable théâtre national québécois avec la représentation des Belles-sœeurs. Il s'agit donc d'un corpus limité mais, comme le signale A. Berman, hautement symbolique. L'auteure indique également qu'elle ne s'interroge pas sur le rôle du traducteur en tant qu'individu mais sur sa fonction sociale ou sur son rôle de porte-parole d'une société. A cette fin elle identifie trois modalités de traduction, iconoclaste, perlocutoire et identitaire, qui correspondent à trois rapports différents entre le texte et sa traduction et à trois procédés différents de sélection et de transformation, c'est-à-dire à trois prises en charge différentes. L'ouvrage s'organise autour de ces trois catégories. Il aurait été utile ici d'établir une différenciation plus nette entre imitation, parodie et adaptation avec des références, ici ou ailleurs, aux travaux de Genette. Ce dernier figure pourtant dans la bibliographie. 
Dans le premier chapitre, A. Brisset détermine le statut de l'Étranger dans l'institution théâtrale au Québec en examinant l'édition des auvres dramatiques en traduction et la programmation étrangère des compagnies théâtrales. Son étude du paratexte met en évidence la préférence de l'adaptation à la traduction ainsi que le biffage quasi total de l'auteur de l'original. Le répertoire québécois comprend donc, par exemple, Lysistrata de Michel Tremblay et L'Effet des rayons gamma sur les vieux garçons de Michel Tremblay et André Brassard, ainsi que La Médée Euripide de Marie Cardinal. Il en est de même en ce qui concerne la programmation théâtrale des ouvres étrangères. Même si on peut constater leur popularité, surtout celle des tragédies modernes américaines et des comédies françaises, il est à noter que, tout comme les traductions publiées, les pièces étrangères représentées sont souvent réappropriées, de sorte que les «cinq pièces de chez nous» du Rideau Vert comprennent une pièce de Tchekov et une d'Albee, ou adaptées; cela met en évidence l'ethnocentrisme du thêâtre québécois. Brisset signale aussi la faible proportion des pièces étrangères autres que celles dont la langue d'origine est l'anglais ou le français. Elle conclut que cette tendance «révèle avec d'autant plus d'éloquence l'autarcie du thêâtre québécois» (p. 85). La sélection des pièces étrangères dépend, semble-t-il, de leur statut canonique et de leur genre, mais la fréquence de leurs représentations est fonction d'un facteur extérieur, le climat social. L'auteure étudie la relation étroite qui existe entre la popularité des pièces authentiquement québécoises et les événements historiques, politiques et sociaux susceptibles d'éveiller les sentiments nationalistes, tels que le référendum. Les chiffres révèlent de façon manifeste la place prédominante occupée par le thêâtre (véritablement) québécois pendant ces moments ainsi que la diminution correspondante accusée par le thêâtre étranger. Cela permet à l'auteure de conclure que «le thêâtre institutionnel apparaît ici comme le miroir de ces événements» (p. 103).

Ayant ainsi constaté l'oblitération de l'altérité aux niveaux de l'édition et de la représentation, Brisset se propose, dans le deuxième chapitre, d'expliquer comment et pourquoi l'œuvre étrangère, pour être acceptée, doit passer pour une ouvre québécoise. Bien que menaçante, l'œuvre de l'Autre fournit au thêatre embryonnaire un modèle nécessaire. Mais, tout en se servant de ce modèle, on peut, en le parodiant, le désintégrer et le transformer en une création québécoise susceptible de contribuer à la fondation d'un thêatre national. Les pièces de Shakespeare, de Brecht, de Molière et même de FélixGabriel Marchand, auteur québécois, subissent donc un transcodage nécessaire à leur réappropriation, car il ne suffit pas de tout simple- 
ment traduire une pièce si on a pour objet d'en faire une création «québécoise» et de «rompre avec la littérature édifiante importée» (p. 117), comme le font Tremblay, Ronfard, Maillet, Germain, Ducharme et Gurik. L'analyse de A Canadian Play / Une plaie canadienne, dont le titre parodie le processus de traduction et la politique de bilinguisme, sert à établir la perception de l'Autre dans la littérature québécoise. Les exemples qui suivent mettent en évidence la façon dont l'Autre est systématiquement éclipsé ou «québécisé». Réécritures plutôt que traductions, ces pièces, versions carnavalisées, joualisées, banalisées, amputées, amplifiées ou enlaidies, ne ressemblent que très peu aux «icônes» dont par contre, comme tous projets parodiants, elles dépendent. Mais dans certains cas le traducteur s'accroche à quelquesuns des «principaux idéologèmes» qui se trouvent dans l'original et qui sous-tendent également le discours sur la condition québécoise; le traducteur en profite pour créer une cuvre «authentiquement» québécoise. Ainsi le sort réservé au «bourgeois gentleman» est celui dont son homologue a été victime: puisque le «gentleman» devient ridicule à cause de son effort de «se faire anglais», sa propre dérision et son discrédit servent à dénigrer «l'Anglais». La version de Maillet s'insère donc dans le discours nationaliste et la pièce de Molière se trouve transformée en «une métaphore pour légitimer subtilement le refus de l'Autre» (p. 140). Il en est de même des autres pièces/traductions/adaptations/travestissements discutés, pour lesquels les cuvres étrangères servent de «matériau de réemploi pour l'édification de la nouvelle dramaturgie québécoise» (p. 191) participant à la quête de l'identité.

La traduction perlocutoire, objet du troisième chapitre, a une fonction persuasive ou incitative qui la rattache à la propagande. L'auteure explique la façon dont Michel Garneau, en «traduisant» Macbeth, le transforme de sorte que la pièce coïncide avec le discours social québécois. La réappropriation d'une pièce anglaise située en Écosse à un moment mythique où le roi d'Angleterre joue le rôle de libérateur pose quand même certains problèmes malgré une intrigue qui présente des affinités avec les idées et les valeurs du «Québec, temps de la réception». L'étude porte sur les changements effectués au niveau de la configuration spatio-temporelle, de la structure et des désignations du «pays» ou de la «nation». Brisset étudie également la mise en valeur chez Garneau de certains thèmes et images tels que ceux de la dépossession collective ou individuelle ou de la plaie ou du sang, interprétants axio-idéologiques qui se trouvent et dans l'œuvre de Shakespeare et dans la jeune littérature québécoise. Grâce à Garneau, Shakespeare, «l'afficheur qui hurle», «le poète nationaliste québécois» non seulement a su contribuer à la nouvelle dramaturgie 
québécoise mais a rejoint sa poésie. Par conséquent, le destin du Québec, si bien représenté dans Macbeth, œuvre iconique, se dote d'un caractère mythique et universel.

Dans le dernier chapitre Brisset, en discutant le statut et les origines de la langue-dialecte québécoise, ouvre à nouveau la boîte de Pandore. Elle s'interroge d'abord sur les origines et le caractère plutôt mythique de la langue et, plus loin, sur la possibilité réelle d'écrire en québécois. Si traduire en québécois veut dire changer, en certains cas, très peu de choses dans le texte et si écrire en québécois veut dire écrire différemment la même séquence à la même page et dans la bouche du même personnage, peut-on vraiment parler d'une langue québécoise d'autant plus que «traduire en québécois», déformation d'ailleurs de la formule parce qu'on indique généralement la langue source ici, est ironique: on dit en français que la langue d'arrivée n'est pas le français. Brisset affirme que les traducteurs qui traduisent en québécois «exercent une fonction plus idéologique que véritablement médiatrice» (p. 309). Ceux-ci ne cherchent pas à «présenter l'œuvre étrangère dans ce qu'elle a de singulier ou d'inédit» (p. 312), mais plutôt à s'en servir afin d'affirmer l'existence et la place légitime de la langue québécoise et sa capacité à transmettre les valeurs et les idées d'une société en quête d'une identité. La rupture avec le français de France contribue donc au projet nationaliste et identitaire.

Mais on aurait tort de croire que l'occultation de l'Autre, la réappropriation et la québécisation du texte sont intrinsèques à la traduction thêâtrale au Québec et que l'autarcie est un fait permanent. Brisset suggère dans la conclusion que cette tendance appartient plutôt aux littératures émergentes: ayant établi sa spécificité nationale, le théâtre québécois peut enfin se permettre d'être universel.

Cette étude qui témoigne d'une recherche minutieuse est avant tout une œuvre critique et ce, comme le signale Berman, dans le sens d'une dénonciation. Provocant et parfois agressif, ce livre ne se veut surtout pas un survol «neutre» de la traduction théâtrale au Québec. Brisset prend comme point de départ que la traduction théâtrale québécoise se donne comme tâche d'intégrer le texte étranger dans son propre discours social ou de lui prêter «le dessein de mettre en scène le fait québécois» (p.312), thèse qu'elle soutient admirablement tout au long de cette étude bien organisée et superbement documentée. Le ton donc est celui d'un débat et l'auteure ne laisse pas de place aux objections. Elle invite plutôt à des répliques (on pourrait proposer par exemple que le spectateur «informé» n'assiste pas à une traduction signée Tremblay sans s'attendre à ce que celui- 
ci laisse glisser le message nationaliste dans le texte). Traducteurs, dramaturges, traductologues et critiques se régaleront donc de cette belle invitation à s'expliquer.

En montrant la façon dont la traduction iconoclaste, perlocutoire et identitaire contribue à l'émergence d'un thêâtre national, l'auteure souligne, comme l'a fait aussi - de façon différente Sherry Simon, la fonction sociale de la traduction et l'importance des contraintes institutionnelles de la société réceptrice. Il faut savoir gré à Annie Brisset d'avoir contribué par cette étude stimulante à cette nouvelle voie dans laquelle la traductologie s'engage.

Jane Koustas Université Brock

Jerzy Tomaszczyk and Barbara Lewandowka-Tomaszczyk, ed. Meaning and Lexicography. Linguistic \& Literary Studies in Eastern Europe (LLSEE), volume 28. Amsterdam/Philadelphia, John Benjamins Publishing Company, 1990, 341 p.

Cet ouvrage réunit des communications présentées à l'Université de Lodz en 1985 lors d'un colloque international de lexicographie et sémantique. Sur invitation, 45 spécialistes s'étaient alors rencontrés pour discuter des différentes approches utilisées actuellement pour aborder les questions sémantiques posées par la lexicologie. Des 27 communications soumises à la discussion, sont aujourd'hui publiés 19 articles (version écrite des communications) dont la diversité est représentative d'une gamme de points de vue engendrée par des cadres théoriques fort différents: grammaire cognitive, sémantique dynamique, grammaire fonctionnelle, modèle Sens-texte, etc. La question du «sens» est ici taillée à facettes de manière à inclure des aspects qui ne sont pas traditionnellement reliés à la sémantique lexicale. Tous les articles sont écrits en anglais et la plupart portent sur l'anglais.

L'entreprise est intéressante et fort louable puisque ces dernières années ont vu le lexique devenir - ou redevenir - un observable digne d'intérêt. Or, le lexique, comme objet d'étude, avait pratiquement été relégué aux oubliettes à la suite du courant linguistique provoqué par le développement du modèle générativiste qui, on 